\title{
Evaluation of Pancreatic Exocrine Function and Zinc Absorption in Alcoholism
}

\author{
HIROYASU IJUIN \\ The Second Department of Medicine, Kurume University School of Medicine, \\ Kurume 830-0011, Japan
}

\begin{abstract}
Summary: Zinc absorption in alcoholism was studied by a combination of zinc tolerance tests in 382 male patients with alcoholism (more than $140 \mathrm{~g} /$ day of ethanol) who had alcoholinduced disease of the liver or pancreas. In study 1 , the serum zinc level was measured in all patients, and serum zinc and fecal chymotrypsin levels were compared in various disease groups. In study 2, 14 patients with liver cirrhosis (LC), 15 with chronic pancreatitis (CP), 7 with $\mathrm{LC}+\mathrm{CP}$, and 7 controls underwent oral zinc tolerance and zinc dipicolinate tolerance tests, zinc absorption and disorders of pancreatic exocrine functions were examined. In study 1, the serum zinc concentration was significantly lower in the CP and LC groups than in the control group, and the fecal chymotrypsin activity was significantly lower in the CP than in the control groups. In study 2, during the oral zinc tolerance test, the serum zinc concentration 3 hours after administration was significantly lower in the LC, CP and LC + CP groups than in the control group. In these groups, the serum zinc concentration was significantly lower in the abnormal fecal chymotrypsin group than in the control group at 2 and 3 hours after administration of zinc sulfate. In the oral zinc dipicolinate tolerance test, the serum zinc levels 2 and 3 hours after administration were significantly elevated in the control and all disease groups; there were no significant differences between the control and each disease group. These results suggest that reduction of pancreatic exocrine functions by alcohol and chronic reduction of synthesis of ligands such as picolinic acid in the liver are involved in the reduction of serum zinc in alcoholism.
\end{abstract}

Key words alcholisn, pancreatic exocrine function, zinc tolerance test, zinc dipicolinate tolerance test

\section{INTRODUCTION}

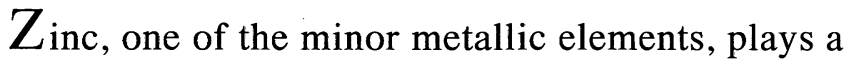
very important role in the maintenance of metabolic functions. A deficiency of zinc causes a growth disorder, dermatopathy, and dysgeusia [1]. Since zinc acts as a coenzyme for carboxypeptidase, one of the pancreatic enzymes [2,3], and is closely related to pancreatic functions, it is considered to be an important factor in digestion and absorption.

Zinc is abundantly present in food and drinking water, and a deficiency of it is rare. However, oligotrophy and abnormalities in digestion and absorption are frequent complications of alcoholism, causing abnormal metabolism of minor elements [4] . Nakamura et al. [5] has reported that a reduction in pancreatic exocrine functions causes a reduction in zinc absorption. Zinc is excreted primarily from the pancreas together with digestive enzymes [6] and is reabsorbed in the intestinal tract by the action of picolinic acid. Picolinic acid is one of substances that enhances absorption of zinc by its strong chelating ability, but the mechanism is unknown [7].

In this study we examined serum zinc levels and zinc absorption using an oral zinc tolerance test in patients with alcohol-induced disorders of the liver 
and pancreas.

\section{SUBJECTS AND METHODS}

We studied 382 male patients with alcoholism (more than $140 \mathrm{~g} /$ day of ethanol) who were hospitalized for withdrawal from alcohol. The mean patient age was $46.5 \pm 11.2$ years. Liver cirrhosis (LC) was present in 166 patients, chronic hepatitis $(\mathrm{CH})$ in 57 patients, hepatic fibrosis (HF) in 94 patients, fatty liver (FL) in 65 patients, and chronic pancreatitis (CP) in 42 patients. Of 42 patients with $\mathrm{CP}, 19$ had LC, 2 had CH, 13 had HF, and 5 had FL. The diagnosis of liver diseases, except clinically obvious LC, was made by liver biopsy, and CP was diagnosed according to the 1983 chronic pancreatitis criteria (JSGE) [8]. The control group comprised 29 normal volunteers.

Serum zinc $(\mathrm{Zn})$ concentrations were measured by atomic absorption spectrophotometry of serum collected immediately after hospitalization. Pancreatic exocrine functions were examined by the fecal chymotrypsin test (FCT) and measured by colorimetric analysis using Monotest Chymotrypsin [9].

\section{Oral zinc tolerance test}

1) In the oral zinc tolerance test, $106 \mathrm{mg}$ of zinc sulfate was orally administered together with $300 \mathrm{ml}$ of water after an overnight fast. The serum $\mathrm{Zn}$ concentration was serially measured before administration and 1, 2 and 3 hours after administration.

2) In the oral zinc dipicolinate tolerance test, 120 $\mathrm{mg}$ of zinc dipicolinate was administered 1 week after the oral zinc tolerance test, and the serum $\mathrm{Zn}$ concentration was serially measured according to the same timetable. Zinc dipicolinate consists of two picolinic acid molecules bound to one zinc atom by chelate bonds [7].

The amount of $\mathrm{Zn}$ in the reagents used in both tolerance tests was $24 \mathrm{mg}$. Informed consent for both the oral zinc and zinc dipicolinate tolerance tests was obtained in writing.

\section{Analysis}

Study 1: The serum $\mathrm{Zn}$ level was measured in all patients to determine whether there were differences in the serum $\mathrm{Zn}$ concentration between disease groups. Pancreatic exocrine functions were similarly evaluated by measuring fecal chymotrypsin activity.

Study 2: The $14 \mathrm{LC}, 15 \mathrm{CP}$, and $7 \mathrm{LC}+\mathrm{CP}$ patients along with 7 controls who underwent the oral zinc tolerance and zinc dipicolinate tolerance tests were also examined for zinc absorption activity to investigate whether there was any correlation between this activity and the disease type or the kind of pancreatic exocrine disorder.

Statistical analysis was performed with Student's $\mathrm{t}$ test, and $\mathrm{P}<.05$ was regarded as significant.

\section{RESULTS}

Evaluation of the serum $\mathrm{Zn}$ concentration and fecal chymotrypsin activity in the various liver and pancreatic disease groups

The serum $\mathrm{Zn}$ concentration was $75.3 \pm 18.7 \mu \mathrm{g} /$ $\mathrm{dl}$ in the CP group, and $72.5 \pm 19.7 \mu \mathrm{g} / \mathrm{dl}$ in the LC

TABLE 1.

The serum $\mathrm{Zn}$ concentration and fecal chymotrypsin activity in the various liver and pancreatic disease groups

\begin{tabular}{ccccccc}
\hline & Control & Chronic Pancreatitis & Liver Cirrhosis & Chronic Hepatitis & Hepatic Fibrosis & Fatty Liver \\
$\mathrm{N}$ & 29 & 42 & 166 & 57 & 94 & 65 \\
\hline $\begin{array}{c}\mathrm{S}-\mathrm{Zn} \\
(\mu \mathrm{g} / \mathrm{dl})\end{array}$ & $88.6 \pm 18.7$ & $75.3 \pm 18.7^{*}(33 \%)$ & $72.5 \pm 19.7^{*}(35 \%)$ & $79.0 \pm 17.7(21 \%)$ & $83.5 \pm 21.9(17 \%)$ & $80.4 \pm 19.4(25 \%)$ \\
$\mathrm{FCH}$ & $50.3 \pm 20.5$ & $28.4 \pm 19.8^{*}(40 \%)$ & $33.2 \pm 26.7(25 \%)$ & $57.1 \pm 33.4(14 \%)$ & $35.4 \pm 22.9(24 \%)$ & $44.1 \pm 30.4(20 \%)$ \\
$(\mathrm{U} / \mathrm{g})$ & & & & & & \\
\hline
\end{tabular}


group, both significantly lower than the control group (88.6 $\pm 14.4 \mu \mathrm{g} / \mathrm{dl})$ (Table 1).

The fecal chymotrypsin activity in the CP group was significantly lower than that in the control $(\mathrm{p}<0.01)$.

Evaluation of the oral zinc tolerance and zinc dipicolinate tolerance tests in the $L C$ and $C P$ groups

After administration of zinc sulfate, the serum zinc concentration was lower in the disease groups than in the control group. Of these disease groups, the $\mathrm{LC}, \mathrm{CP}$ and $\mathrm{LC}+\mathrm{CP}$ groups showed significantly lower serum zinc concentration than the control group at 2 hours after administration (Fig. 1a).

There was no significant difference in the serum zinc concentration between zinc sulfate and zinc dipicolinate in measurements obtained 1 hour after administration, while those obtained 2 and 3 hours after administration showed significant elevation of

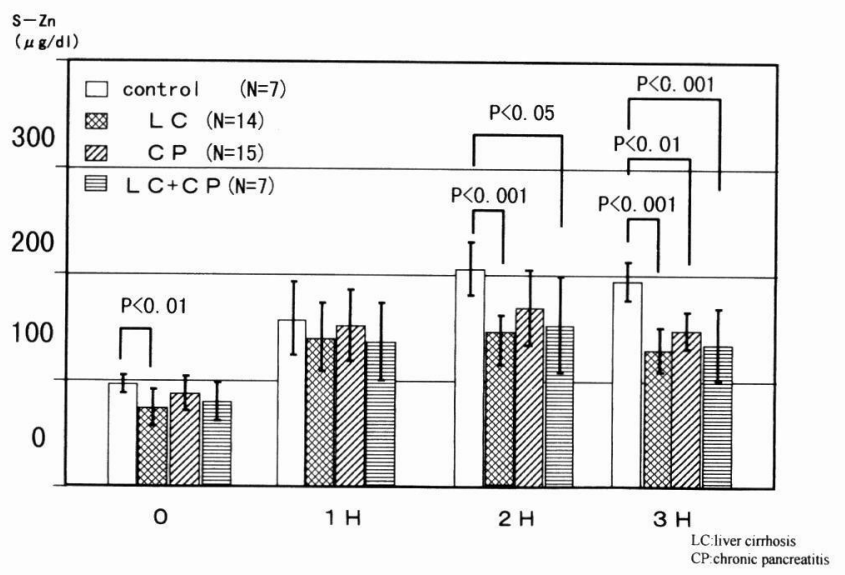

Fig. 1a. The oral zinc tolerance tests in the liver cirrhosis and chronic pancreatitis groups.

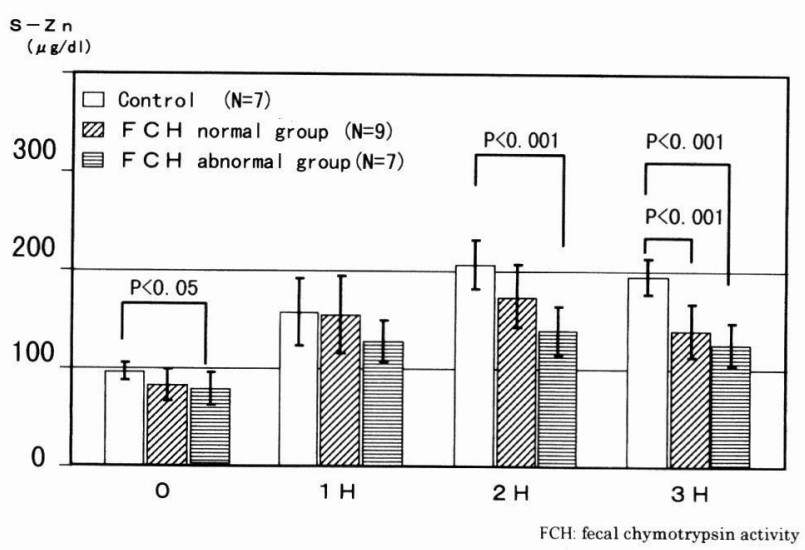

Fig. 2a. The oral zinc tolerance tests in the normal and abnormal fecal chymotrypsin subgroups of liver cirrhosis patients. the serum zinc concentration in all disease groups. There were no significant differences between controls and any of the disease groups (Fig. 1b).

Evaluation of the oral zinc tolerance and zinc dipicolinate tolerance tests in the normal and abnormal fecal chymotrypsin subgroups of $L C$ patients

During the oral zinc tolerance test the serum zinc concentration was lower in both the normal and abnormal fecal chymotrypsin groups than in the control group. The serum zinc was significantly lower in the abnormal fecal chymotrypsin group than in the control group 2 hours after zinc sulfate administration. Three hours after administration, the serum zinc was significantly lower in the normal fecal chymotrypsin group and in the abnormal fecal chymotrypsin group than in the control group (Fig. $2 a)$.

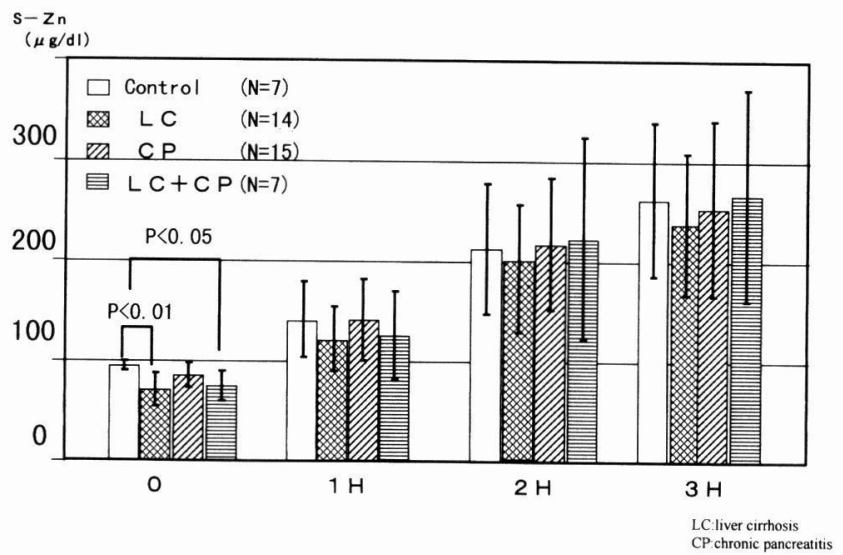

Fig. 1b. The oral zinc dipicolinate tolerance tests in the liver cirrhosis and chronic pancreatitis groups.

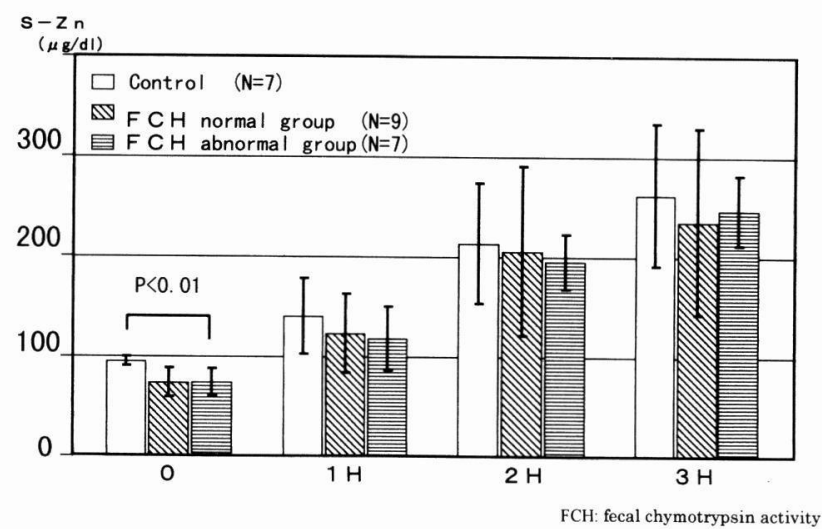

Fig. $2 b$. The oral zinc dipicolinate tolerance tests in the normal and abnormal fecal chymotrypsin subgroups of liver cirrhosis patients. 
There was no significant difference in the serum zinc concentration between zinc sulfate and zinc dipicolinate measured 1 hour after administration. At 2 and 3 hours after administration of zinc dipicolinate, the serum zinc concentration was significantly elevated in all disease groups, and there were no significant differences between the control and any of the disease groups (Fig. 2b).

Evaluation of the oral zinc tolerance and zinc dipicolinate tolerance tests in the normal and abnormal fecal chymotrypsin subgroups of $C P$ patients

During the oral zinc tolerance test, the serum zinc concentration was lower in the $\mathrm{CP}$ group than in the control group. It was significantly lower in the abnormal fecal chymotrypsin group than in the control group at 2 and 3 hours after administration of zinc sulfate (Fig. 3a).

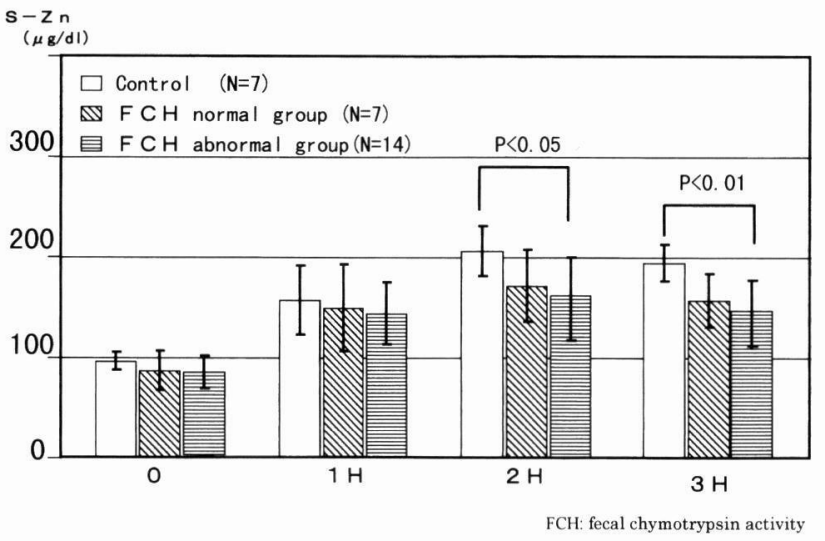

Fig. 3a. The oral zinc tolerance tests in the normal and abnormal fecal chymotrypsin subgroups of chronic pancreatitis patients.

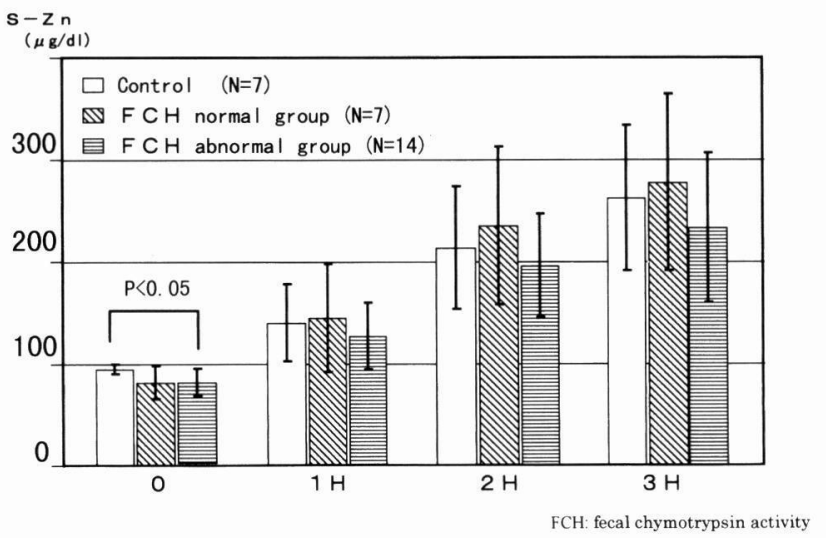

Fig. 3b. The oral zinc dipicolinate tolerance tests in the normal and abnormal fecal chymotrypsin subgroups of chronic pancreatitis patients.
There was no significant difference in the serum zinc level between zinc sulfate and zinc dipicolinate if measured 1 hour after their administration, while at 2 and 3 hours after administration of zinc dipicolinate, the serum zinc concentration was significantly elevated in all disease groups, and there was no significant difference between the control and each disease group (Fig. 3b).

\section{DISCUSSION}

Insufficient intake of zinc in alcoholism is considered to be due to an unbalanced diet [4]. In this study, however, the serum zinc concentration was significantly lower in the LC and CP patients. This result cannot be explained solely by insufficient intake of zinc because zinc is present in drinking water as well as food. The results of the oral zinc tolerance test showed that zinc absorption ability was significantly lower in the LC and CP groups than in the control group. The serum zinc concentration in LC patients is considered to be reduced by enhancement of zinc excretion in the urine $[10,11]$, reduction of binding proteins in the serum [12], and reduction of absorption in the gastrointestinal tract [12-14]. Unlike patients with viral LC, many patients with alcoholic LC have pancreatic disorders even though they do not satisfy the diagnostic criteria for $\mathrm{CP}$. These patients were classified into the normal and abnormal groups according to the fecal chymotrypsin level, and the serum zinc level was examined. There was no significant difference between the two groups, but there was a greater difference between the control group and the abnormal group than between the control group and normal group. Similar results were obtained for the CP patients. Reduction of the serum zinc concentration in alcoholism was more often observed in the abnormal fecal chymotrypsin group than in the normal fecal chymotrypsin group, suggesting that it is related to the reduction of pancreatic exocrine function. The mechanism of reduction of the serum zinc level by reduction of pancreatic exocrine function may be that zinc absorption is reduced by the decrease of picolinic acid secreted into the intestine. The mechanism of the action of picolinic acid is unknown, but it is thought that picolinic acid is produced from tryptophan in the liver and kidney, secreted with the pancreatic juice into the intestine, and bound to $\mathrm{Zn}$ to generate zinc dipicolinate, thus enhancing the absorption of zinc [7].

Based on the results of animal experiments, 
Suzuki et al. [13] have proposed that zinc absorption is divided into two phases; diffusion and active transport $[15,16]$. Transport of zinc within 1 hour after administration is considered to be due to diffusion because of the concentration difference between the interior and exterior of the intestine. Thereafter, active transport by carriers such as histidine and picolinic acid becomes the major pathway of zinc transportation. In the present study, the serum zinc concentration was significantly lower in both CP and LC groups than in the control group only on measurements obtained 2 and 3 hours after administration. However, there were no differences between the control and the CP and LC groups in serum zinc concentration measured 2 and 3 hours after administration of zinc dipicolinate, suggesting that chelated zinc was actively absorbed. In LC patients without reduction of pancreatic exocrine function, zinc absorption was also improved by administration of zinc dipicolinate. This suggests that the reduction of serum zinc in patients with alcoholic $\mathrm{LC}$ is caused by a reduction of synthesis of picolinic acid in the liver in addition to the potential disorder of pancreatic exocrine functions.

These results suggest that the alcohol-induced reduction of pancreatic exocrine function and chronic reduction of ligands such as picolinic acid synthesized in the liver are involved in the reduction of serum zinc in alcoholism.

ACKNOWLEDGMENTS: The author would like to thank Prof. Kyuichi Tanikawa and Dr. Hirohiko Abe for their critical advice and encouragement during the course of this study.

\section{REFERENCES}

1. Prasad AS, Halsted JA, and Nadimi M. Syndrome of iron deficiency anemia, hepatosplenomegaly and geophagia. Am J Med 1961; 31:532-546.

2. Mills CF, Quarterman J, Williams RB, and Dargarno AC. The effect of zinc deficiency on pancreatic car- boxypeptidase activity and protein and absorption in the rat. Biochem 1967; 102:712-718.

3. Vallee BL, and Neurath H. Carboxipeptidase, a zinc metalloenzyme. J Biol Chem 1955; 217:253-261.

4. Dinsmore WW, McMaster D, Callender ME, Buchanan $\mathrm{KD}$, and Love AHG. Trace element and alcohol. Sci Total Environ 1985; 42:109-119.

5. Nakamura K. Experimental study on zinc metabolism and pancreatic function in the fibrotic pancreas after pancreatic duct ligation in dog. J JPN PANC SOC 1991; 6:393-405.

6. Gjorup I, Petronijevic L, Rubinstein E, Anderson B, Worning $\mathrm{H}$ et al. Pancreatic secretion of zinc and copper in normal subjects and in patients with chronic pancreatitis. Digestion 1991; 49:161-166.

7. Evance GW. Normal and abnormal zinc absorption in man and animals: The tryptphan connection. Nutr Rev 1980; 38:137-141.

8. Chronic Pancreatitis Examination Committee. Chronic pancreatitis criteria. Jap J Gastroent 1983; 80:1863-1866.

9. Fukaya Y, Miyakawa K, Masaki M, Yamauchi H, and Satou T. Clinical value of fecal chymotripsin test for the evaluation of pancreatic exocrine function. J Bil Panc 1985; 6:1137-1143.

10. Lindeman RD, Baxter DJ, and Yunice AA. Serum concentration and urinary excretions zinc in cirrhosis, nephrotic syndrome and renal insufficiency. Am J Med Sci 1978; 275:17-31.

11. Walker BE, Dawson JB, Kelleher J, and Losowsky MS. Plasma and urinary zinc in patients with malabsorption syndromes or hepatic cirrhosis. Gut 1973; 14:943-948.

12. Chen S. Hypo zincemia in compensated cirrhotic patients. Yokohama Igaku 1991; 42:307-314. (in Japanese)

13. Suzuki K, Arakawa Y, Matuo Y, Matuo H, and Takeuchi S. Disturbance of the intestinal absorption of trace metals, especially zinc in the patients with chronic liver diseases. Digestion \& Absorption 1988; 11:51-54.

14. Herruzo AS, Cuenca DE, and Rivero M. Intestinal zinc absorption in cirrhotic patients. J Gastenterol 1989; 27: 335-338.

15. Davis NT. Studies on the absorption of zinc by rat intestine. Br J Nutr 1980; 43:189-203.

16. Lee HH, Prasad AS, Brewer GJ, and Owyang C. Zinc absorption in human small intestine. Am J Physiol 1989; 19:G87-G91. 